\title{
Short-term mortality following surgical procedures for the diagnosis of pediatric brain tumors: outcome analysis in 5533 children from SEER, 2004-2011
}

\author{
Todd C. Hankinson, MD, MBA, ${ }^{1,3}$ Roy W. R. Dudley, MD, PhD, ${ }^{2}$ Michelle R. Torok, PhD, ${ }^{3}$ \\ Mohana Rao Patibandla, MCh, ${ }^{1}$ Kathleen Dorris, MD, ${ }^{4}$ Seerat Poonia, BA, ${ }^{5}$ C. Corbett Wilkinson, MD, ${ }^{1}$ \\ Jennifer L. Bruny, MD, ${ }^{6}$ Michael H. Handler, MD, ${ }^{1}$ and Arthur K. Liu, MD, PhD ${ }^{7}$ \\ ${ }^{5}$ University of Colorado School of Medicine; ${ }^{1}$ Pediatric Neurosurgery and ${ }^{4}$ Division of Neuro-oncology, Department of Pediatrics; \\ ${ }^{6}$ Pediatric Surgery, Department of Surgery, University of Colorado School of Medicine, Children's Hospital Colorado; ${ }^{3}$ Adult and \\ Child Center for Health Outcomes Research; ' ${ }^{7}$ Department of Radiation Oncology, University of Colorado Anschutz Medical \\ Campus, Aurora, Colorado; and 'Division of Neurosurgery, Department of Pediatric Surgery, Montreal Children's Hospital, McGill \\ University Health Centers, Montreal, Quebec, Canada
}

OBJECTIVE Thirty-day mortality is increasingly a reference metric regarding surgical outcomes. Recent data estimate a 30-day mortality rate of 1.4\%-2.7\% after craniotomy for tumors in children. No detailed analysis of short-term mortality following a diagnostic neurosurgical procedure (e.g., resection or tissue biopsy) for tumor in the US pediatric population has been conducted.

METHODS The Surveillance, Epidemiology and End Results (SEER) data sets identified patients $\leq 21$ years who underwent a diagnostic neurosurgical procedure for primary intracranial tumor from 2004 to 2011. One- and two-month mortality was estimated. Standard statistical methods estimated associations between independent variables and mortality.

RESULTS A total of 5533 patients met criteria for inclusion. Death occurred within the calendar month of surgery in 64 patients $(1.16 \%)$ and by the conclusion of the calendar month following surgery in 95 patients $(1.72 \%)$. Within the first calendar month, patients $<1$ year of age $(n=318)$ had a risk of death of $5.66 \%$, while those from 1 to 21 years $(n=5215)$ had a risk of $0.88 \%(p<0.0001)$. By the end of the calendar month following surgery, patients $<1$ year $(n=318)$ had a risk of death of $7.23 \%$, while those from 1 to 21 years $(n=5215)$ had a risk of $1.38 \%(p<0.0001)$. Children $<1$ year at diagnosis were more likely to harbor a high-grade lesion than older children (OR 1.9, 95\% Cl 1.5-2.4).

CONCLUSIONS In the SEER data sets, the risk of death within 30 days of a diagnostic neurosurgical procedure for a primary pediatric brain tumor is between $1.16 \%$ and $1.72 \%$, consistent with contemporary data from European populations. The risk of mortality in infants is considerably higher, between $5.66 \%$ and $7.23 \%$, and they harbor more aggressive lesions.

http://thejns.org/doi/abs/10.3171/2015.7.PEDS15224

KEY WORDS 30-day mortality; pediatric brain tumor; Surveillance, Epidemiology and End Results; oncology

$\mathrm{E}$ CONOMIC forces in health care combined with data availability have led to increased scrutiny of outcomes regarding clinical care. ${ }^{2,9,15}$ Entities that assess quality, such as US News and World Report and the American College of Surgeons National Quality Improvement Program (ACS NSQIP), employ metrics of shortterm outcome, such as mortality or return to the operat- ing room within 30 days. ${ }^{3,14}$ As a measure of short-term surgical outcomes, 30-day mortality rate offers the advantage of temporal proximity to the intervention in question. This provides both ease of data collection and the intuitive sense of a direct relationship between the intervention and the outcome. In the absence of benchmark data, however, the value of a given measurement may be difficult to as-

ABBREVIATIONS ACS NSQIP = American College of Surgeons National Quality Improvement Program; AT/RT = atypical teratoid/rhabdoid tumor; GTR = gross-total resection; ICD-O-3 = International Classification of Diseases for Oncology, 3rd Edition; NIS = Nationwide Inpatient Sample; NOS = not otherwise specified; NRTC = National Registry of Childhood Tumors; PNET = primitive neuroectodermal tumor; SEER = Surveillance, Epidemiology and End Results; STR = subtotal resection.

SUBMITTED April 10, 2015. ACCEPTED July 13, 2015.

INCLUDE WHEN CITING Published online November 20, 2015; DOI: 10.3171/2015.7.PEDS15224. 
sess. In addition, accurate understanding of the mortality risk that is faced by children with complex disease contributes substantially to the optimization of surgical and multidisciplinary management plans.

European population-based studies recently reported all-cause 30-day mortality rates following craniotomy for pediatric brain tumors to be $1.4 \%-2.7 \% .^{14,17}$ These studies acknowledge that such low rates limit the statistical power of the analyses. O'Kane and colleagues ${ }^{14}$ also noted a substantially higher 30-day mortality rate in the UK population, when compared with the Norwegian population ( $2.7 \%$ versus $1.4 \%$ ), but were not able to explain this difference. This uncertainty prompted us to explore whether short-term mortality rates were well established in the US population. A study of the Nationwide Inpatient Sample (NIS) demonstrated an in-hospital mortality rate of $1.6 \%$ following craniotomy for resection of pediatric brain tumors. ${ }^{16}$ However, there has been no study examining the overall (i.e., inpatient and outpatient) short-term mortality rate following diagnostic neurosurgical procedures (e.g., resection or biopsy) for pediatric brain tumors in the US. As such, this project was undertaken to help determine a range of short-term mortality rates following the diagnosis of pediatric brain tumors in the US, and to determine if any specific presenting characteristics were associated with elevated rates of mortality.

The National Cancer Institute's Surveillance, Epidemiology and End Results (SEER) program includes data regarding approximately $28 \%$ of the US population and is widely considered to be the most reliable and authoritative source of population-based cancer statistics in the US. ${ }^{12,13}$ This makes SEER an excellent resource for the study of phenomena that may have a low frequency, such as shortterm mortality following surgery. We used the SEER data sets to help establish a baseline short-term mortality rate following diagnostic neurosurgical procedures for tumor in children in the US. We further analyzed the data for characteristics that correlate with an elevated risk of mortality at this time point.

\section{Methods}

The sample frame was identified from the most recent SEER data sets (Incidence - SEER 18 Regs Research Data + Hurricane Katrina Impacted Louisiana Cases, Nov 2012 Sub [1973-2011 varying]; http://seer.cancer.gov/data/seerstat/nov2012/). We included patients $\leq 21$ years diagnosed with 1 of 80 tumors that present most frequently in the intracranial compartment (Table 1). The sample was further restricted to tumors located within 1 of the 12 intracranial anatomical sites in SEER. These included categories corresponding to each of the cerebral lobes (frontal, temporal, parietal, and occipital, as well as cerebrum, cerebellum, brainstem, cerebral meninges, ventricles, optic nerves, brain-not otherwise specified (NOS), and overlapping lesion of the brain). ${ }^{1}$ Spinal cord tumors were not included. Lastly, the sample was restricted to those patients who were treated with a surgical procedure that was consistent with craniotomy or tumor biopsy. These were then divided into 2 groups: subtotal resection (STR) and gross-total resection (GTR), using previously published methods. ${ }^{6,710}$ Briefly, STR included patients with surgical codes 20 (lo- cal excision of tumor, lesion, or mass; excisional biopsy; or stereotactic biopsy of the brain) and 21 (subtotal resection of tumor, lesion or mass in brain), while GTR included patients with surgical codes 30 (radical, total, gross resection of tumor, lesion or mass in brain), 40 (partial resection of lobe of brain, when the surgery cannot be coded as 20-30), and 55 (GTR of lobe of brain [lobectomy]). The data set was limited to cases diagnosed beginning in 2004 because prior to this time point SEER did not include data regarding patients with nonmalignant lesions.

Independent variables included sex, age at diagnosis, race, extent of resection, anatomical location of tumor, and date of diagnosis. For most analyses we categorized age as $<1,1-4,5-9,10-14$, and 15-19 years. Due to an apparent survival disadvantage among younger patients, we dichotomized age into $<1$ year versus $\geq 1$ year to examine agespecific survival. Race was initially divided into 4 categories, according to SEER: "White," "Black," "Other," and "Unknown." For survival analysis, the variable was dichotomized as "White" and "Black/Other." The "Unknown" category was excluded from these analyses, leaving a total of 4197 subjects for analyses regarding race (Table 2). The histological grade of tumors was taken from SEER. Tumors with a histological subcode of " 3 " were considered to be "high grade" and those with subcodes " $/ 0$ " and "/1" were considered "low grade." The only exception to this was code "9421/3: Pilocytic Astrocytoma, malignant," which encompasses all pilocytic astrocytomas. Although pilocytic astrocytomas generally do not display malignant behavior and were changed from malignant behavior ("/3") to borderline behavior ("/1") in the ICD-O-3, SEER registrars were instructed to continue to assign the code " $/ 3$ " for pilocytic astrocytomas to maintain data recording consistency. ${ }^{14}$ The subcode " $/ 2$ " applies to carcinoma in situ and thus does not apply to brain tumors.

The outcome, mortality, was based on the "vital status recode" variable and was defined as death from any cause. We calculated mortality according to the method of Welch and Black. ${ }^{20}$ Briefly, SEER does not report cancerdirected surgery dates, but does report month and year of diagnosis and death. It requires the date of diagnosis to precede or coincide with cancer-directed therapy and considers surgery to be a cancer-directed therapy. Therefore, patients who died of any cause within the month of diagnosis also died within the same month as surgery. Limiting analysis to this patient group would, however, systematically underestimate the 30-day postoperative mortality rate, as the diagnostic surgical procedure could occur on any day of the month in question. We therefore also calculated the rate of death at the end of the calendar month following diagnosis. This method alone could include patients who survived for up to 61 days following surgery. The combination of two calculations, however, establishes a range within which lies the true value for 30-day mortality. Therefore, this range estimates the 30-day mortality.

Data were analyzed with SAS version 9.3 and SEER*Stat 8.1.2 (http://seer.cancer.gov/seerstat/). Crude probability of death using expected survival was calculated using the actuarial method. Bivariable comparisons were tested using chi-square tests. Crude and adjusted odds ratios and 95\% confidence intervals were estimated with logistic regres- 
TABLE 1. Frequency of assessed tumors in the total sample and children younger than 1 year at diagnosis*

\begin{tabular}{|c|c|c|c|c|}
\hline \multirow[b]{2}{*}{ ICD-O-3: Histological Behavior } & \multicolumn{2}{|c|}{ All Patients $(n=5533)$} & \multicolumn{2}{|c|}{ Patients <1 Yr $(n=318)$} \\
\hline & No. & $\%$ & No. & $\%$ \\
\hline 9421/3: Pilocytic astrocytoma, malignant $†$ & 1285 & 23.22 & 39 & 12.26 \\
\hline 9470/3: Medulloblastoma, NOS & 566 & 10.23 & 20 & 6.29 \\
\hline 9505/1: Ganglioglioma, NOS & 362 & 6.54 & 9 & 2.83 \\
\hline 9400/3: Astrocytoma, NOS & 241 & 4.36 & 19 & 5.97 \\
\hline 9440/3: Glioblastoma, NOS & 225 & 4.07 & 15 & 4.72 \\
\hline 9391/3: Ependymoma, NOS & 214 & 3.87 & 8 & 2.52 \\
\hline 9392/3: Ependymoma, anaplastic & 194 & 3.51 & 18 & 5.66 \\
\hline 9473/3: Primitive neuroectodermal tumor & 190 & 3.43 & 13 & 4.09 \\
\hline 9350/1: Craniopharyngioma & 168 & 3.04 & 4 & 1.26 \\
\hline 9508/3: Atypical teratoid/rhabdoid tumor & 143 & 2.58 & 49 & 15.41 \\
\hline 9413/0: Dysembryoplastic neuroepithelial tumor & 140 & 2.53 & 0 & 0.00 \\
\hline 9560/0: Neurilemmoma, NOS & 130 & 2.35 & 0 & 0.00 \\
\hline 9450/3: Oligodendroglioma, NOS & 119 & 2.15 & 1 & 0.31 \\
\hline 9401/3: Astrocytoma, anaplastic & 107 & 1.93 & 5 & 1.57 \\
\hline 9390/0: Choroid plexus papilloma, NOS & 106 & 1.92 & 24 & 7.55 \\
\hline 9380/3: Glioma, malignant & 105 & 1.90 & 4 & 1.26 \\
\hline 9351/1: Adamantinomatous craniopharyngioma & 100 & 1.81 & 1 & 0.31 \\
\hline 9424/3: Pleomorphic xanthoastrocytoma & 96 & 1.74 & 0 & 0.00 \\
\hline 9064/3: Germinoma & 94 & 1.70 & 0 & 0.00 \\
\hline 9471/3: Desmoplastic medulloblastoma & 84 & 1.52 & 5 & 1.57 \\
\hline 9382/3: Mixed glioma & 77 & 1.39 & 4 & 1.26 \\
\hline 9530/0: Meningioma, NOS & 73 & 1.32 & 1 & 0.31 \\
\hline 9384/1: Subependymal giant cell astrocytoma & 69 & 1.25 & 2 & 0.63 \\
\hline 9420/3: Fibrillary astrocytoma & 59 & 1.07 & 2 & 0.63 \\
\hline 9161/1: Hemangioblastoma & 57 & 1.03 & 1 & 0.31 \\
\hline 9474/3: Large cell medulloblastoma & 54 & 0.98 & 0 & 0.00 \\
\hline 9390/3: Choroid plexus papilloma, malignant & 46 & 0.83 & 19 & 5.97 \\
\hline 9506/1: Central neurocytoma & 45 & 0.81 & 0 & 0.00 \\
\hline 9362/3: Pineoblastoma & 39 & 0.70 & 3 & 0.94 \\
\hline 9412/1: Desmoplastic infantile astrocytoma & 32 & 0.58 & 20 & 6.29 \\
\hline 9539/1: Atypical meningioma, NOS & 24 & 0.43 & 1 & 0.31 \\
\hline 9531/0: Meningothelial meningioma & 23 & 0.42 & 1 & 0.31 \\
\hline 9085/3: Mixed germ cell tumor & 22 & 0.40 & 0 & 0.00 \\
\hline 9505/3: Ganglioglioma, anaplastic & 22 & 0.40 & 0 & 0.00 \\
\hline 9451/3: Oligodendroglioma, anaplastic & 19 & 0.34 & 1 & 0.31 \\
\hline 9080/3: Teratoma, malignant, NOS & 18 & 0.33 & 8 & 2.52 \\
\hline 9390/1: Atypical choroid plexus papilloma & 17 & 0.31 & 6 & 1.89 \\
\hline 9492/0: Gangliocytoma & 15 & 0.27 & 2 & 0.63 \\
\hline 9441/3: Giant cell glioblastoma & 14 & 0.25 & 0 & 0.00 \\
\hline 9080/0: Teratoma, benign & 13 & 0.23 & 1 & 0.31 \\
\hline 9383/1: Subependymoma & 11 & 0.20 & 0 & 0.00 \\
\hline 9537/0: Transitional meningioma & 11 & 0.20 & 0 & 0.00 \\
\hline 9361/1: Pineocytoma & 8 & 0.14 & 0 & 0.00 \\
\hline 9430/3: Astroblastoma & 7 & 0.13 & 1 & 0.31 \\
\hline 9532/0: Fibrous meningioma & 7 & 0.13 & 0 & 0.00 \\
\hline 9150/1: Hemangiopericytoma, NOS & 6 & 0.11 & 3 & 0.94 \\
\hline 9534/0: Angiomatous meningioma & 6 & 0.11 & 0 & 0.00 \\
\hline 9490/3: Ganglioneuroblastoma & 5 & 0.09 & 0 & 0.00 \\
\hline
\end{tabular}


TABLE 1. Frequency of assessed tumors in the total sample and children younger than 1 year at diagnosis* (continued)

\begin{tabular}{|c|c|c|c|c|}
\hline \multirow[b]{2}{*}{ ICD-0-3: Histological Behavior } & \multicolumn{2}{|c|}{ All Patients $(n=5533)$} & \multicolumn{2}{|c|}{ Patients <1 Yr $(n=318)$} \\
\hline & No. & $\%$ & No. & $\%$ \\
\hline 9493/0: Dysplastic gangliocytoma of cerebellum & 5 & 0.09 & 1 & 0.31 \\
\hline 9530/3: Meningioma, malignant & 5 & 0.09 & 1 & 0.31 \\
\hline 9100/3: Choriocarcinoma & 4 & 0.07 & 1 & 0.31 \\
\hline 9411/3: Gemistocytic astrocytoma & 4 & 0.07 & 1 & 0.31 \\
\hline 9533/0: Psammomatous meningioma & 4 & 0.07 & 0 & 0.00 \\
\hline 9538/1: Clear cell meningioma & 4 & 0.07 & 0 & 0.00 \\
\hline 9538/3: Papillary meningioma & 4 & 0.07 & 0 & 0.00 \\
\hline 9150/3: Hemangiopericytoma, malignant & 3 & 0.05 & 2 & 0.63 \\
\hline 9352/1: Papillary craniopharyngioma & 3 & 0.05 & 0 & 0.00 \\
\hline 9393/3: Papillary ependymoma, NOS & 3 & 0.05 & 0 & 0.00 \\
\hline 9444/1: Chordoid glioma & 3 & 0.05 & 1 & 0.31 \\
\hline 9490/0: Ganglioneuroma & 3 & 0.05 & 0 & 0.00 \\
\hline 9560/3: Neurilemmoma, malignant & 3 & 0.05 & 0 & 0.00 \\
\hline 9070/3: Embryonal carcinoma, NOS & 2 & 0.04 & 0 & 0.00 \\
\hline 9071/3: Yolk sac tumor & 2 & 0.04 & 0 & 0.00 \\
\hline 9080/1: Teratoma, NOS & 2 & 0.04 & 0 & 0.00 \\
\hline 9081/3: Teratocarcinoma & 2 & 0.04 & 1 & 0.31 \\
\hline 9370/3: Chordoma, NOS & 2 & 0.04 & 0 & 0.00 \\
\hline 9442/1: Gliofibroma & 2 & 0.04 & 0 & 0.00 \\
\hline 9442/3: Gliosarcoma & 2 & 0.04 & 0 & 0.00 \\
\hline 9150/0: Hemangiopericytoma, benign & 1 & 0.02 & 0 & 0.00 \\
\hline 9410/3: Protoplasmic astrocytoma & 1 & 0.02 & 0 & 0.00 \\
\hline 9472/3: Medullomyoblastoma & 1 & 0.02 & 0 & 0.00 \\
\hline
\end{tabular}

* Shaded tumors are the 5 most common in children younger than 1 year.

$\dagger$ WHO Grade I pilocytic astrocytoma.

sion. Statistical tests were 2-tailed and $\mathrm{p}$ values of $<0.05$ were considered statistically significant.

\section{Results}

\section{Mortality Within the Same Month as Diagnosis}

A total of 5533 patients met criteria for inclusion in the analysis (Table 1). Death occurred within the same calendar month as diagnosis in 64 patients $(1.16 \%)$. Patients younger than 1 year $(\mathrm{n}=318)$ had a risk of death of $5.66 \%(\mathrm{n}=18)$ while those diagnosed from ages 1 to 21 years $(n=5215)$ had a risk of death of $0.88 \%(n=46, p$ $<0.0001$ ). The crude odds ratio of death among children younger than 1 year versus older children was 6.7 (95\% CI 3.9-11.8). Children 10-15 years of age experienced the lowest crude mortality rate, $0.42 \%$ (Table 2 ). Among all children younger than 16 years, the observed rate of death was $1.29 \%(54 / 4187)$.

Patients of nonwhite race had a risk of death of $1.4 \%$, which was higher than the risk for those of white race (1.1\%), but this did not reach statistical significance $(\mathrm{p}=$ 0.46 ). No difference was identified in outcome based on year of diagnosis, sex, or extent of resection. After adjusting for sex, race, and categorized year of diagnosis, variables associated with mortality within the month of diagnosis were age less than 1 year at diagnosis and high-grade tumor histology (Table 3, c-statistic 0.72).

\section{Mortality Before the End of the Calendar Month Following Diagnosis}

Death occurred before the end of the calendar month following diagnosis in 95 patients (1.72\%), which includes the 64 patients who died during the same month as diagnosis. Patients younger than 1 year $(n=318)$ had a risk of death of $7.23 \%(n=23)$, while those $1-21$ years $(n=5215)$ had a risk of $1.38 \%(\mathrm{n}=72, \mathrm{p}=<0.0001)$. The crude odds ratio of death among children younger than 1 year of age versus older children was 5.57 (95\% CI 3.43-9.04). As with mortality within the first calendar month, children 10-15 years of age experienced the lowest crude rate, $0.56 \%$ (data not shown). Among all children younger than 16 years, the observed rate of death was $1.89 \%(79 / 4187)$. No differing trends were identified with analysis of death within 2 months of diagnosis when compared with death within 1 month of diagnosis.

\section{Histological Characteristics}

In the entire data set, the 5 most common tumors were pilocytic astrocytoma $(\mathrm{n}=1285,23.22 \%)$; medulloblastoma, NOS $(\mathrm{n}=566,10.23 \%)$; ganglioglioma, $\operatorname{NOS}(\mathrm{n}=$ $362,6.54 \%)$; astrocytoma, NOS $(\mathrm{n}=241,4.36$ ); and glioblastoma, NOS ( $\mathrm{n}=225,4.07 \%)$ (Table 1). Among patients less than 1 year of age, the 5 most common tumors were atypical teratoid/rhabdoid tumor $(\mathrm{AT} / \mathrm{RT})(\mathrm{n}=49$, 
TABLE 2. Association between demographic/clinical characteristics and death within the first calendar month*

\begin{tabular}{|c|c|c|c|c|c|}
\hline Variable & Total $(n=5533)$ & Dead $(n=64)$ & Alive $(n=5469)$ & p Value & Crude OR $(95 \% \mathrm{Cl})$ \\
\hline Sex & & & & $0.80(\mathrm{P})$ & \\
\hline Male & $3026(54.7)$ & $36(1.2)$ & $2990(98.8)$ & & Reference \\
\hline Female & $2507(45.3)$ & $28(1.1)$ & $2479(98.9)$ & & $0.94(0.57-1.54)$ \\
\hline Dichotomized race $(n=5428)$ & & & & $0.46(P)$ & \\
\hline White & $4432(81.7)$ & $50(1.1)$ & $4382(98.9)$ & & Reference \\
\hline Black/other & $996(18.3)$ & $14(1.4)$ & $982(98.6)$ & & $1.25(0.69-2.27)$ \\
\hline Categorized age (yrs) & & & & $<0.0001(\mathrm{P})$ & \\
\hline 0 & $318(5.7)$ & $18(5.7)$ & $300(94.3)$ & & $8.02(3.66-17.54)$ \\
\hline $1-4$ & $1229(22.2)$ & $22(1.8)$ & $1207(98.2)$ & & $2.44(1.15-5.16)$ \\
\hline $5-9$ & $1213(21.9)$ & $8(0.7)$ & $1205(99.3)$ & & $0.89(0.35-2.26)$ \\
\hline $10-15$ & $1427(25.8)$ & $6(0.4)$ & $1421(99.6)$ & & $0.56(0.20-1.56)$ \\
\hline $16-21$ & $1346(24.3)$ & $10(0.7)$ & $1336(99.3)$ & & Reference \\
\hline Dichotomized age (yrs) & & & & $<0.0001(F)$ & \\
\hline$\geq 1$ & $5215(94.3)$ & $46(0.9)$ & $5169(99.1)$ & & Reference \\
\hline$<1$ & $318(5.7)$ & $18(5.7)$ & $300(94.3)$ & & $6.74(3.86-11.77)$ \\
\hline Tumor grade & & & & $<0.0001(\mathrm{P})$ & \\
\hline Low grade & $2888(52.2)$ & $16(0.6)$ & $2872(99.4)$ & & Reference \\
\hline High grade & 2645 (47.8) & $48(1.8)$ & 2597 (98.2) & & $3.32(1.88-5.86)$ \\
\hline Type of surgery & & & & $0.33(\mathrm{P})$ & \\
\hline STR & 1695 (30.6) & $16(0.9)$ & 1679 (99.1) & & Reference \\
\hline GTR & $3838(69.4)$ & $48(1.3)$ & $3790(98.7)$ & & $1.33(0.75-2.35)$ \\
\hline Yr of diagnosis & & & & $0.10(P)$ & \\
\hline 2004 & $688(12.4)$ & $16(2.3)$ & $672(97.7)$ & & $2.55(0.99-6.56)$ \\
\hline 2005 & $658(11.9)$ & $8(1.2)$ & $650(98.8)$ & & $1.32(0.46-3.82)$ \\
\hline 2006 & $687(12.4)$ & $6(0.9)$ & 681 (99.1) & & $0.94(0.30-2.94)$ \\
\hline 2007 & 707 (12.8) & $6(0.8)$ & 701 (99.2) & & $0.92(0.29-2.86)$ \\
\hline 2008 & $743(13.4)$ & $11(1.5)$ & $732(98.5)$ & & $1.61(0.59-4.38)$ \\
\hline 2009 & $711(12.9)$ & $6(0.8)$ & 705 (99.2) & & $0.91(0.29-2.84)$ \\
\hline 2010 & $690(12.5)$ & $5(0.7)$ & $685(99.3)$ & & $0.78(0.24-2.58)$ \\
\hline 2011 & $649(11.7)$ & $6(0.9)$ & $643(99.1)$ & & Reference \\
\hline
\end{tabular}

$\mathrm{F}=$ Fisher's exact test; $\mathrm{P}=$ Pearson's chi-square test.

* Unless otherwise indicated, values are numbers (\%) of patients.

$15.41 \%)$; pilocytic astrocytoma $(n=39,12.26 \%)$; choroid plexus papilloma, NOS $(\mathrm{n}=24,7.55 \%)$; medulloblastoma, NOS $(n=20,6.29 \%)$; and desmoplastic infantile astrocytoma $(n=20,6.29 \%)$. The odds ratio for death within the first calendar month among those with a high-grade tumor was 2.9 (95\% CI 1.6-5.1) when compared with those with a low-grade lesion when adjusting for sex, race, age and year of diagnosis (Table 3). It should be noted that due to inherent subjectivity in the process, we did not collapse entities with multiple SEER ICD-O-3 codes (e.g., Ependymoma, NOS, 9391/3, and Ependymoma, anaplastic, 9392/3) into a single group. As such, the frequency of a given histological entity may not appear to be consistent with well-established data.

\section{Children $<1$ Year at Diagnosis}

There were 318 patients $<1$ year at the time of diagnosis (Tables 2 and 4). Among these children, there was no difference in sex distribution, race, extent of resection, or year of diagnosis between those who died and those who did not. Infants were more likely than older children to present with a high-grade tumor (OR 1.9, 95\% CI 1.5-2.4, Table 5). Regarding infants who died within the calendar month of diagnosis, there was a nonsignificant difference in the histological grade of lesions, with infants who died having OR 3.2 (95\% CI 0.9-11.1) of presenting with a high-grade tumor relative to a low-grade tumor.

There was no statistically significant difference in sex distribution, extent of resection, or year of diagnosis between patients who were less than or older than 1 year at the time of diagnosis. There was, however, a statistically significant difference in the neuroanatomical distribution of tumors between those who were diagnosed before and after their first birthday (data not shown). In those children less than 1 year, more tumors were located in the ventricle (23.0\% vs $8.6 \%$ ) and brain/NOS (16.7\% vs $9.3 \%)$, while in patients $\geq 1$ year, more tumors were located in the cerebel$\operatorname{lum}(24.1 \%$ vs $15.1 \%)$. 
TABLE 3. Adjusted associations between demographic and clinical characteristics and 1-month mortality in 5428 patients*

\begin{tabular}{cc}
\hline \multicolumn{1}{c}{ Variable } & Adjusted OR $(95 \% \mathrm{Cl})$ \\
\hline Sex & Reference \\
\hline Female & $1.1(0.6-1.8)$ \\
\hline Male & Reference \\
\hline Race & $1.2(0.6-2.2)$ \\
\hline White & \\
\hline Black/other & $5.9(3.4-10.4)$ \\
\hline Dichotomized age in yrs & Reference \\
\hline$\leq 1$ & \\
\hline $1-21$ & $1.3(0.8-2.1)$ \\
\hline Yr of diagnosis & Reference \\
\hline $2004-2007$ & \\
\hline $2008-2011$ & $2.89(1.6-5.1)$ \\
\hline Tumor grade & Reference \\
\hline High & \\
\hline Low & \\
\hline$*$
\end{tabular}

\section{Discussion}

The SEER data sets indicate that, in the US, the rate of 30-day mortality following a diagnostic neurosurgical procedure for brain tumor in the pediatric population is between $1.16 \%$ and $1.72 \%$. This rate is substantially lower than that reported by O'Kane and colleagues from the UK's National Registry of Childhood Tumors (NRCT), which was approximately $2.7 \%$ between 2004 and 2007.14 Consistent with our findings regarding patients in the US, Smith and colleagues used the NIS to identify an inhospital mortality rate of $1.2 \%$ between 1997 and $2000 .{ }^{16}$ Among children less than 16 years of age at diagnosis, the rate of death in SEER is $1.29 \%-1.93 \%$. This is very simi-
TABLE 5. Tumor grade and patient age at diagnosis

\begin{tabular}{|c|c|c|c|c|}
\hline \multirow[b]{2}{*}{ Variable } & \multicolumn{2}{|c|}{ Age of Child* } & \multirow[b]{2}{*}{ p Value } & \multirow[b]{2}{*}{$\begin{array}{c}\text { Crude OR } \\
(95 \% \mathrm{Cl})\end{array}$} \\
\hline & $\begin{array}{c}<1 Y r \\
(n=318)\end{array}$ & $\begin{array}{c}\geq 1 Y r \\
(n=5215)\end{array}$ & & \\
\hline High-grade tumor & 199 (62.6) & $2446(46.9)$ & $<0.0001$ & $1.9(1.5-2.4)$ \\
\hline Low-grade tumor & $119(37.4)$ & 2769 (53.1) & & \\
\hline
\end{tabular}

* Data are presented as number of patients (\%).

lar to the rate of $1.4 \%$ reported for the same age group by Solheim and colleagues ${ }^{17}$ using data from the Norwegian cancer registry in patients treated after the 1990s. In their study, there was no difference in perioperative mortality between patients younger than 16 years and those between 16 and 69 years, but these authors did not subdivide the pediatric population by age. The current study is the largest reported examination of short-term mortality rates following a diagnostic neurosurgical procedure for children with brain tumors. It is also the first such study of the US population. The factors that correlated with 30-day mortality were age at diagnosis and histological grade of the brain tumor. Year of diagnosis, sex, race, and extent of diagnosis did not correlate with 30-day mortality.

\section{Age at Diagnosis}

Our data demonstrate significantly higher odds of short-term mortality (OR 6.7) in children younger than 1 year at diagnosis versus children 1-21 years of age. A similar result was seen in the study by O'Kane and colleagues, in which children less than 2 years of age had a 30 -day mortality rate of $7.1 \%$ versus $2.0 \%$ for children 2-14 years of age, which was also statistically significant. ${ }^{14}$ These 30 -day mortality findings, showing worse outcome for infants, help explain the results of previous work, which showed elevated longer-term mortality rates for infants with brain tumors. Lang and colleagues re-

TABLE 4. Association between demographic/clinical characteristics and death within the first calendar month in 318 patients younger than 1 year*

\begin{tabular}{|c|c|c|c|c|}
\hline Variable & Total $(n=318)$ & Dead $(n=18)$ & Alive $(n=300)$ & $\mathrm{p}$ Value \\
\hline Sex & & & & $0.87(\mathrm{P})$ \\
\hline Male & $165(51.9)$ & $9(50.0)$ & $156(52.0)$ & \\
\hline Female & $153(48.1)$ & $9(50.0)$ & $144(48.0)$ & \\
\hline Dichotomized race $(n=310)$ & & & & $1.00(\mathrm{~F})$ \\
\hline White & $251(81.0)$ & $15(83.3)$ & $236(80.8)$ & \\
\hline Black/Other & $59(19.0)$ & $3(16.7)$ & $56(19.2)$ & \\
\hline Tumor grade & & & & $0.06(P)$ \\
\hline Low grade & $119(37.4)$ & $3(16.7)$ & $116(38.7)$ & \\
\hline High grade & $199(62.6)$ & $15(4.7)$ & $184(61.3)$ & \\
\hline \multicolumn{5}{|l|}{ Type of surgery } \\
\hline STR & $93(29.3)$ & $4(22.2)$ & $89(29.7)$ & $0.50(\mathrm{P})$ \\
\hline GTR & $225(70.8)$ & $14(77.8)$ & $211(70.3)$ & \\
\hline Categorized yr of diagnosis & & & & $0.67(\mathrm{P})$ \\
\hline $2008-2011$ & $157(49.4)$ & $8(44.4)$ & $149(49.7)$ & \\
\hline 2004-2007 & $161(50.6)$ & $10(55.6)$ & $151(50.3)$ & \\
\hline
\end{tabular}

* Unless otherwise indicated, values are numbers of patients (\%). 
ported a 1-year survival of 53\% among their 16 patients who were treated with craniotomy for brain tumor before 6 months of age. ${ }^{11}$ In their series, among the 8 patients who did not survive the follow-up period, the median time to death was 3.9 months from surgery, and 2 patients died intraoperatively. More recently, Mathew et al., compared outcomes for children with brain tumors recorded in the SEER data sets to those recorded in the NRTC from the UK. ${ }^{12}$ These authors found 1-, 5-, and 10-year survival rates for children less than 3 years of age to be $81.1 \%$, $70.6 \%$, and $68.8 \%$ in the SEER data sets and $76.9 \%$, $57.0 \%$, and $51.4 \%$ in the NRTC, while children 3-14 years old had survival rates of $90.4 \%, 79.7 \%$, and $78.2 \%$ in SEER and $86.5 \%, 72.9 \%$, and $68.8 \%$ in NRCT. In both data sets young children did worse than older children, and again, like the 30-day data presented here compared with O'Kane and colleagues' study, outcomes were slightly better from the SEER data sets than the NRCT. ${ }^{12,14}$ It is unclear why the 30-day mortality rate would be different between the UK and the US. As O'Kane and colleagues have stated previously, this might be a statistical anomaly because of the relatively small number of outcome events (i.e., deaths) that occur in the first 30 days after surgery. ${ }^{14}$ However, other potential reasons for differences must be considered, such as possible differences in time to presentation, surgical technique, perioperative care, and/or management of adjuvant therapies.

The challenges of performing craniotomy and brain tumor resection in very young children have been well documented. ${ }^{11,18,19}$ These include the expected high rate of intraoperative blood loss relative to low total circulating blood volume, the immature and friable brain parenchyma and blood vessels, difficulties with perioperative thermoregulation in very young children, and limitations regarding head fixation of the immature skull. Furthermore, due to the open anterior fontanel and cranial sutures, brain tumors in very young children can grow to a very large size, and are often not recognized until the children present with macrocephaly and a bulging fontanel, or eventually decompensate with irritability, poor feeding, vomiting, and lethargy. ${ }^{11,18,19}$ Although adjuvant therapies would rarely contribute to death within 30 days of surgery, the options for neoadjuvant treatment after minimally invasive biopsy may be limited in infants. Cranial irradiation in young children is associated with unacceptable adverse effects on multiple levels, including neurocognitive development, growth delay, and secondary malignancies; therefore, such therapy is usually not offered to children less than 3 years of age. ${ }^{8,18}$ Chemotherapy is a very effective treatment after surgery for many pediatric brain tumors, but it too can be associated with significant morbidity related to febrile neutropenia, as well as hematopoetic and gastrointestinal toxicities in infants. ${ }^{5}$

An elevated risk of short-term complications following surgical procedures in the very young, including mortality, is not limited to the neurosurgical population. Using beta-phase data from the American College of Surgeons National Surgical Quality Program (ACS NSQIP) Pediatric program, Bruny and colleagues ${ }^{4}$ reported higher rates of 30-day mortality following surgical procedures in neonates, relative to all cases, in general surgery, orthopedics, otolaryngology, and neurosurgery. Among the neurosurgery cohort, the 30-day mortality rate in neonates was $1.4 \%$ versus $0.7 \%$ in children of all ages. In the pediatric surgery cohort, the 30 -day mortality rate in neonates was $3.2 \%$ versus $0.6 \%$ in children of all ages.

\section{Tumor Histology in Children of All Ages}

In the SEER data sets, high-grade tumor histology correlated with worse 30 -day mortality rates in children, with an odds ratio of death of 3.3 for high-grade versus lowgrade tumors. O'Kane et al. also reported higher 30-day mortality rates for children with malignant brain tumors, the highest being $18.8 \%$ for choroid plexus carcinomas, $13 \%$ for "embryonal tumors other than medulloblastoma" (which incorporated other primitive neuroectodermal tumors [PNETs] and AT/RTs), and 10\% for high-grade astrocytoma. ${ }^{14}$ All other tumor types had 30 -day mortality rates less than 5\%. While Solheim et al. did not stratify their 30-day mortality data for children by histological groups, they did so for their entire population, and found that the 4 highest mortality rates were for parenchymal pineal region tumors $(6.7 \%)$, choroid plexus tumors (5.8\%), PNET/medulloblastoma (5.3\%), and mixed/high-grade astrocytoma $(5.4 \%){ }^{17}$ The former 3 tumor types are much more common in children than in adults, again suggesting that worse 30-day outcomes in children are associated with highergrade tumor histology.

\section{Tumor Histology in Very Young Children}

Very young children also more frequently present with brain tumors of higher histological grade..$^{11,18}$ In the SEER data sets, infants were more likely than older children to present with high-grade tumors (OR 1.9, 95\% CI 1.5-2.4; Table 5). Indeed, in SEER the most common tumor in children less than 1 year of age was AT/RT (15.41\%). Likewise, in the study by Lang et al., which queried a single center's (Children's Hospital of Philadelphia) database for all brain tumor resections in children less than 6 months of age, the most common tumors were AT/RT and PNET, each of which accounted for approximately $19 \%$ of all cases, and all of these patients died within 18 months after diagnosis. ${ }^{11}$ In the SEER data sets there was a trend toward children under 1 year of age with high-grade tumors having a higher 30-day mortality rate than children of similar age who had low-grade tumors, but this did not achieve statistical significance, perhaps due to the small numbers of patients in comparison groups when subdivided in this manner. Very young children also had twice as many intraventricular tumors than older children $(23.0 \%$ vs $8.6 \%)$, while older children had more cerebellar tumors than the younger children $(24.1 \%$ vs $15.1 \%)$. Ventricular location can complicate the surgical treatment by making such tumors less accessible to resection, allowing dissemination via CSF pathways, and causing hydrocephalus either by blocking CSF pathways or by increasing CSF production in the case of choroid plexus tumors. Unfortunately, SEER does not provide information on metastasis at presentation or hydrocephalus, which is a limitation of the use of this data set for 30-day outcomes research. 


\section{Limitations}

Limitations of the SEER data sets for outcomes analysis have been well described by our group and others. ${ }^{6,7,10,20}$ While often relevant when regarding studies of benign lesions, the short duration of available follow-up in SEER is not a limitation to this study, which is focused only on 1-2 month follow-up. The absence of a specific code indicating the date of surgery limits the precision of this study. As described by Welch and Black, ${ }^{20}$ however, SEER rules regarding the assignment of diagnosis prior to (or concurrent with) a therapeutic intervention, provide the opportunity to estimate the rate of death, as we have done. Despite the fact that one of SEER's strengths is the large sample size, our statistical analysis was limited by the relatively small numbers of outcome events (i.e., death). Indeed, among 5533 pediatric patients with brain tumors, there were 64 the end of the same month as surgery and 95 deaths by by the end of the calendar month after surgery. Another potential limitation of this study pertains to the assumption that 30-day mortality is directly or indirectly related to the surgery itself. For the calculation of mortality, we chose to use all-cause, rather than tumor-specific, mortality. This was based on the a priori belief that it was appropriate to include any mortality occurring within a short time frame following surgery, regardless of the documented cause of death. This position is substantiated by Solheim and colleagues ${ }^{17}$ who reported that less than $5 \%$ of death certificates recorded complication from surgery as a factor in the cause of death in their series.

\section{Conclusions}

In the SEER data sets, the crude probability of death within 30 days of a diagnostic neurosurgical procedure for a primary pediatric brain tumor is between $1.16 \%$ and $1.72 \%$, which is consistent with population-based data from Norway, but lower than that reported through the UK's NRCT. The risk of short-term mortality is influenced by patient age at diagnosis and histological tumor grade. In particular, infants are at considerably higher risk of 30-day mortality after a diagnostic tumor procedure. While these patients harbor more histologically aggressive lesions, it is also known that surgical intervention in such children is particularly challenging for a variety of reasons. Specific efforts should be undertaken to identify methods to minimize the risk of perioperative and short-term mortality in this most susceptible group.

\section{Acknowledgments}

Special thanks go to Judy Gault, $\mathrm{PhD}$, for her assistance in reviewing and editing this manuscript.

\section{References}

1. Adamo MB, Johnson CH, Ruhl JL, Dickie LA (eds): 2012 SEER Program Coding and Staging Manual (NIH Publication Number 12-5581). Bethesda, MD: National Cancer Institute, 2012

2. American Board of Medical Specialties: Steps Toward Initial Certification and MOC. (http://www.abms.org/ board-certification/steps-toward-initial-certification-and$\mathrm{moc} /$ ) [Accessed September 2, 2015]
3. Borzecki AM, Christiansen CL, Chew P, Loveland S, Rosen AK: Comparison of in-hospital versus 30-day mortality assessments for selected medical conditions. Med Care 48:1117-1121, 2010

4. Bruny JL, Hall BL, Barnhart DC, Billmire DF, Dias MS, Dillon PW, et al: American College of Surgeons National Surgical Quality Improvement Program Pediatric: a beta phase report. J Pediatr Surg 48:74-80, 2013

5. Chi SN, Gardner SL, Levy AS, Knopp EA, Miller DC, Wisoff JH, et al: Feasibility and response to induction chemotherapy intensified with high-dose methotrexate for young children with newly diagnosed high-risk disseminated medulloblastoma. J Clin Oncol 22:4881-4887, 2004

6. Dudley RWR, Torok MR, Gallegos D, Liu AK, Handler MH, Hankinson TC: Pediatric choroid plexus tumors: epidemiology, treatments, and outcome analysis on 202 children from the SEER database. J Neurooncol 121:201-207, 2015

7. Dudley RWR, Torok MR, Gallegos DR, Mulcahy-Levy JM, Hoffman LM, Liu AK, et al: Pediatric low-grade ganglioglioma: epidemiology, treatments, and outcome analysis on 348 children from the surveillance, epidemiology, and end results database. Neurosurgery 76:313-320, 2015

8. Duffner PK, Krischer JP, Horowitz ME, Cohen ME, Burger PC, Friedman HS, et al: Second malignancies in young children with primary brain tumors following treatment with prolonged postoperative chemotherapy and delayed irradiation: a Pediatric Oncology Group study. Ann Neurol 44:313-316, 1998

9. Hammers R, Anzalone S, Sinacore J, Origitano TC: Neurosurgical mortality rates: what variables affect mortality within a single institution and within a national database? $\mathbf{J}$ Neurosurg 112:257-264, 2010

10. Hankinson TC, Fields EC, Torok MR, Beaty BL, Handler $\mathrm{MH}$, Foreman NK, et al: Limited utility despite accuracy of the national SEER dataset for the study of craniopharyngioma. J Neurooncol 110:271-278, 2012

11. Lang SS, Beslow LA, Gabel B, Judkins AR, Fisher MJ, Sutton LN, et al: Surgical treatment of brain tumors in infants younger than six months of age and review of the literature. World Neurosurg 78:137-144, 2012

12. Mathew RK, O'Kane R, Parslow R, Stiller C, Kenny T, Picton $S$, et al: Comparison of survival between the UK and US after surgery for most common pediatric CNS tumors. Neuro Oncol 16:1137-1145, 2014

13. McGuire CS, Sainani KL, Fisher PG: Both location and age predict survival in ependymoma: a SEER study. Pediatr Blood Cancer 52:65-69, 2009

14. O'Kane R, Mathew R, Kenny T, Stiller C, Chumas P: United Kingdom 30-day mortality rates after surgery for pediatric central nervous system tumors. J Neurosurg Pediatr 12:227-234, 2013

15. Shaw K, Cassel CK, Black C, Levinson W: Shared medical regulation in a time of increasing calls for accountability and transparency: comparison of recertification in the United States, Canada, and the United Kingdom. JAMA 302:20082014, 2009

16. Smith ER, Butler WE, Barker FG II: Craniotomy for resection of pediatric brain tumors in the United States, 1988 to 2000: effects of provider caseloads and progressive centralization and specialization of care. Neurosurgery 54:553565,2004

17. Solheim O, Jakola AS, Gulati S, Johannesen TB: Incidence and causes of perioperative mortality after primary surgery for intracranial tumors: a national, population-based study. J Neurosurg 116:825-834, 2012

18. Souweidane MM: Brain tumors in the first two years of life, in Albright A, Pollack I, Adelson P (eds): Principles and Practice of Pediatric Neurosurgery. New York: Thieme, 2014 
19. Teo WY, Myseros JS: The gut or the brain?-Gastrointestinal misdiagnoses of infantile brain tumors. Childs Nerv Syst 30:1449-1453, 2014

20. Welch HG, Black WC: Are deaths within 1 month of cancerdirected surgery attributed to cancer? J Natl Cancer Inst 94:1066-1070, 2002

\section{Disclosures}

Colorado Clinical and Translational Sciences Institute/Children's Hospital Colorado Research Institute KL2 Research Scholar Award (NCATS/NIH UL1 TR001082) (to T.C.H.), and Morgan Adams Foundation (T.C.H.), NCI Paul Calabresi Career Development Award for Clinical Oncology (K12CA086913) (to K.D.).

\section{Author Contributions}

Conception and design: Hankinson, Dudley, Torok, Poonia, Wilkinson, Handler, Liu. Acquisition of data: Hankinson, Torok, Poonia. Analysis and interpretation of data: Hankinson, Dudley, Torok, Liu. Drafting the article: Hankinson, Dudley, Patibandla. Critically revising the article: all authors. Reviewed submitted version of manuscript: all authors. Approved the final version of the manuscript on behalf of all authors: Hankinson. Statistical analysis: Torok. Study supervision: Hankinson, Liu.

\section{Correspondence}

Todd C. Hankinson, Pediatric Neurosurgery, Children's Hospital Colorado, University of Colorado Anschutz Medical Campus, Aurora, CO 80045. email: todd.hankinson@ childrenscolorado. org. 